\title{
A long term study of axonal transport in the central visual system following eye enucleation in the adult cat
}

\author{
M.-J. Pinilla-Morillo*, D. Sanides**, and K. Albus \\ Abteilung für Neurobiologie, Max-Planck-Institut für Biophysikalische Chemie, Am Faßberg, D-3400 Göttingen, \\ Federal Republic of Germany
}

Summary. The effect of the enucleation of one eye on anterograde and retrograde labelling in geniculo-cortical, cortico-geniculate and commissural projections was investigated in adult cats by means of horseradish peroxidase (HRP) and tritiated aminoacids. It was found that in addition to the immediate decrease of retrograde labelling with HRP in the cortical projections from the deafferented A-laminae of the dorsal part of the lateral geniculate nucleus (Singer et al. 1977) there is a further reduction which lasts up to 75 days after enucleation. At 146 and 363 days after enucleation a slight increase in the number of labelled neurones was noted in the deafferented lamina. Qualitative assessment did not reveal any changes of anterograde labelling with tritiated amino acids in geniculo-cortical, cortico-geniculate and commissural axones. In addition, the retrograde labelling with HRP in cortico-geniculate and commissural projections seemed to be unaffected by eye enucleation.

Key words: Eye enucleation - Geniculo-cortical and cortico-geniculate axones - Axonal transport - HRP - Amino acids

In the adult cat, immediately after enucleation of one eye, retrograde labelling with horseradish peroxidase (HRP) diminishes noticeably in contralateral geniculo-cortical neurones (Singer et al. 1977). The immediate decrease in retrograde labelling is most probably due to decreased endocytotic up-

\footnotetext{
* Present address: Dept. of Anatomy, Medical Faculty, University of Zaragoza, Zaragoza, Spain

** Deceased

Offprint requests to: $\mathrm{K}$. Albus (address see above)
}

take of HRP into thalamo-cortical terminals (Holtzman et al. 1971; Schacker et al. 1974; Enerbäck et al. 1980) caused by the suppression of neuronal activity in deafferented geniculate neurones (Sakakura and Iwama 1967; Eysel and Grüsser 1975). These geniculo-cortical neurones show transneuronal atrophy at later times after eye enucleation (Cook et al. 1951; Guillery 1973). This suggests possible alterations in tracer uptake which might be utilized to demonstrate ocular dominance patches, not only in geniculo-cortical afferents (Shatz et al. 1977; Ito et al. 1977) but in other central visual projections as well. To test this assumption we have investigated anterograde and retrograde labelling in geniculo-cortical, cortico-geniculate and commissural projections of the visual cortex (areas 17, 18 and 19) at various times after eye enucleation using HRP and tritiated amino acids as tracer substances.

This report is based on the results from 25 adult cats. Enucleation of one eye and the injection of tracers were performed under pentobarbitone anaesthesia (Nembutal ${ }^{\circledR} 35 \mathrm{mg} / \mathrm{kg}$ i.p.). The interval between enucleation and injection of the tracer was between $2 \mathrm{~h}$ and 12 month. The exact age of the cats at the time of eye enucleation was not known, but all animals were certainly older than 10 month. The animals received injections of either HRP (Boehringer Grade 1; 30\% in Ringer solution, $0.1-0.4 \mu \mathrm{l}$ per injection) or an equal mixture of $4.5-{ }^{3} \mathrm{H}$-Leucine and $5-{ }^{3} \mathrm{H}$-Proline (dissolved in Ringer solution, $60 \mu \mathrm{Ci} / \mu \mathrm{l})$. The tracers were injected by pressure via glass pipettes (tip diameter about $40 \mu \mathrm{m}$ ) into the lateral gyrus of one hemisphere or into one or both LGNds. The cortical injection sites comprised the areas 17, 18 and 19. The position of the LGNd was monitored by recording the activity of single units or unit clusters with a tungsten-in-glass microelectrode fixed in 
parallel to the injection pipette. After the injection the animals survived $2 \mathrm{~d}$ (after HRP injections) or $3 \mathrm{~d}$ (after Leucine/Proline injections). Then they were given an overdose of pentobarbitone and perfused through the heart with Ringer solution followed by fixative (after HRP injections 1\% paraformaldehyde, $2 \%$ glutardialdehyde and $5 \%$ sucrose in $0.1 \mathrm{M}$ cacodylate buffer $\mathrm{pH} 7.2$; after Leucine/Proline injections 4\% formalin).

After HRP injections, the injection sites were separated from the sites of origin of a projection. Frozen sections of $60 \mu \mathrm{m}$ thickness in a frontal or horizontal plane (injection sites) and frontal or parasagittal plane (thalamus) were cut. Every 5th section of the injection site and every section of the LGNd region was incubated according to the Graham \& Karnovsky's method (1966). The sections were subsequently mounted, dehydrated, cleared and coverslipped, and were examined under the light microscope at a final magification of $\times 800$ (oil immersion). The positions of all labelled neurones were plotted with a X-Y plotter coupled to the microscope stage. We counted only neurons in which the nucleus could be clearly identified. Then the coverslips were removed, the sections were rehydrated and counterstained with $0.1 \%$ thionine for the exact identification of the LGNd layers. Finally, all labelled neurones in lamina A were counted separately from those in lamina $A 1$, from the position plots. The number of HRP labelled neurons in the deafferented lamina (either A or A1) was calculated as a percentage of all labelled neurones in both laminae $\mathrm{A}$ and A1.

After Leucine/Proline injections blocks containing the injection site and the projection targets were embedded in paraplast. Sections of $10 \mu \mathrm{m}$ thickness were cut in a parasagittal plane. Every 5 th section was mounted and prepared for autoradiography (Ilford $\mathrm{K} 2$ emulsion; exposure time 4 weeks). The developed sections were counterstained with $0.1 \%$ thionine, dehydrated, cleared and coverslipped. Terminal fields were examined and photographed under darkfield illumination.

Immediately after enucleation of one eye, the number of retrogradely labelled neurones in the deafferented layer of the LGNd, contralateral to enucleation, was considerably reduced (Fig. 1). The proportion of labelled neurones in the deafferented layer $\mathrm{A}$ of the two cases investigated was $42 \%$ and $25 \%$, respectively. The respective proportion in two control cases, i.e. in nonoperated animals, was $48 \%$ and $51 \%$. The proportion of labelled neurones was $28 \% 35$ days after enucleation, this decreased to about $16 \%$ about 75 days

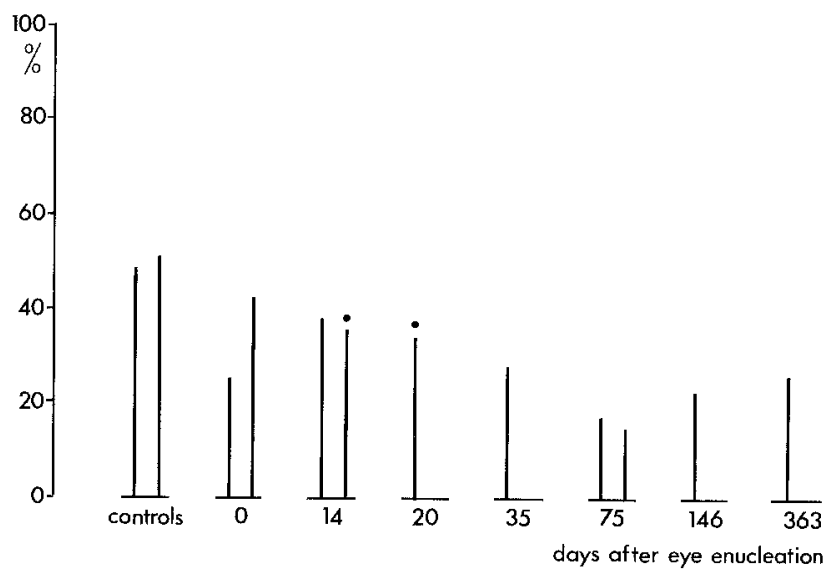

Fig. 1. Retrograde labelling with HRP of neurones in the LGNd following eye enucleation. The bar indicates the number of HRP labelled neurones in the deafferented lamina (A or A1) as a percentage of all labelled neurones in both laminae $A$ and A1. Each bar represents the result from one animal. Neurones were counted in the LGNd contralateral (bars) or ipsilateral (bars with point on top) to eye enucleation. The number of sections analyzed and the number of neurones counted was as follows: Controls, (1st): $31 / 4351 ;$ (2nd) $16 / 1834 ; 0$ days (1st) 53/1218; (2nd) 22/302; 14 days, contra, 42/578; ipsi, 18/492; 20 days, ipsi, $11 / 799$; 35 days $35 / 2224 ; 75$ days (1st) $35 / 784$; (2nd) $26 / 364 ; 146$ days $39 / 1350 ; 363$ days $75 / 5632.146$, and 363 days after eye enucleation neurons in the deafferented laminae appeared to be smaller than neurons in the intact laminae; quantitative measurements of cell size were not performed

after enucleation. Six or twelve months after enucleation there was a slight increase in the proportion of labelled neurones to about $24 \%$. The reduction in the number of labelled neurones in the deafferented lamina of the ipsilateral LGNd which is moderate immediately after eye enucleation (Singer et al. 1977) is even more clearly seen after 14-20 days (Fig. 1).

Eye enucleation does not seem to affect labelling via the other central visual projections tested in this experiment. The anterograde labelling of tritiated amino acids from the cortex back to the LGNd was tested in 3 cases and, as shown in Fig. 2, the density of labelled terminals is about the same in the normal and the deafferented lamina of various times after enucleation. Continuous bands of label were also seen in the contralateral visual cortex, around the $17 / 18$ border, and at the outer border of area 19. Retrograde labelling in cortico-geniculate neurones was tested by injecting HRP into laminae A and A1 of the contralateral eye of two cases, 20 days after eye enucleation. The retrogradely labelled neurones in layer VI of area $17 / 18$ and 19 were homogeneously distributed and no patches indicating possibly an ocular dominance organization could be seen (not shown here). In two of the experiments with cortical injections 

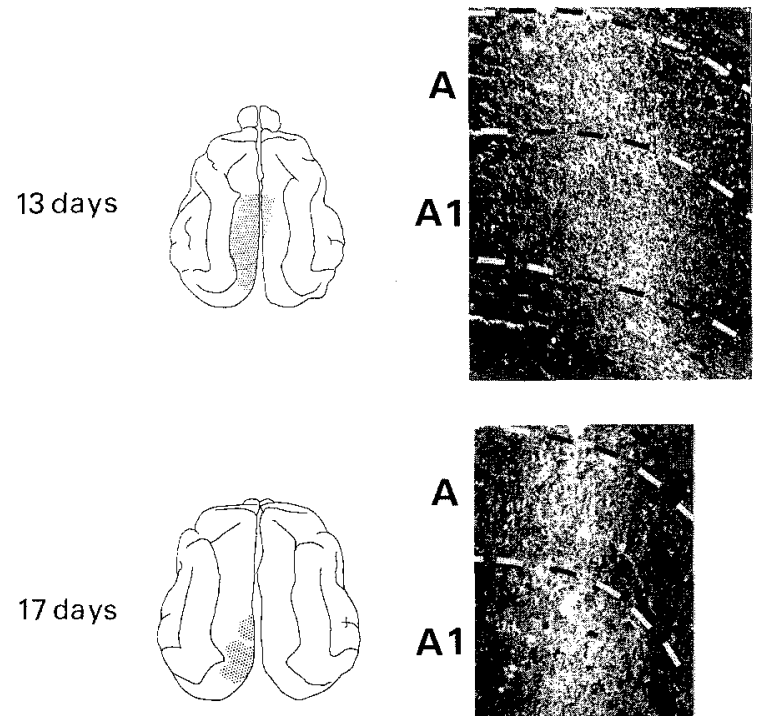

A

A1
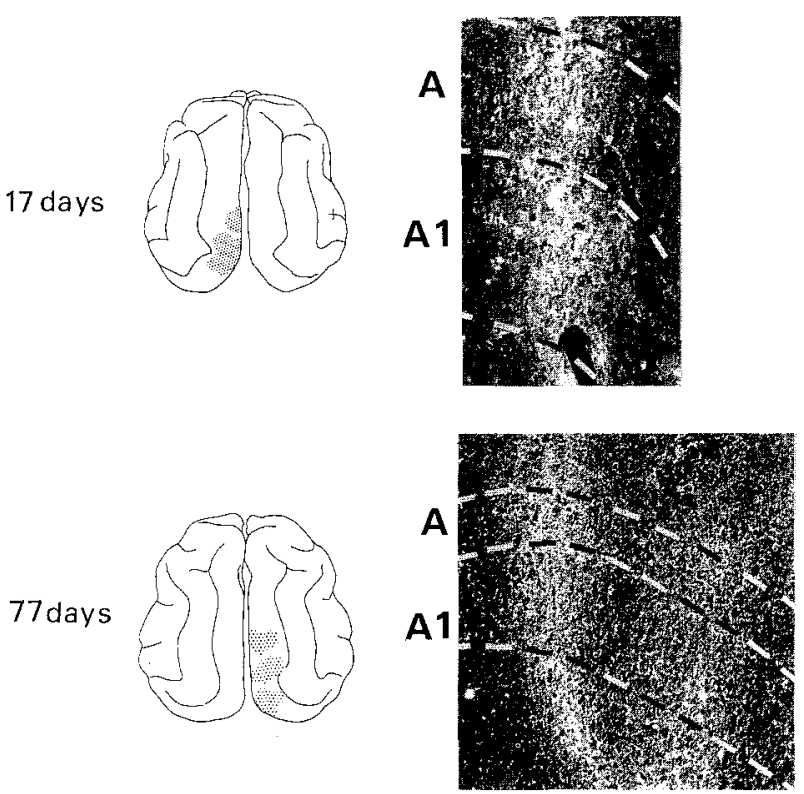

Fig. 2. Anterograde labelling in corticogeniculate projection following eye enucleation. Sections through the LGNd with anterogradely labelled terminal fields are reproduced in darkfield illumination in the right column. The borders of the laminae $\mathrm{A}$ and $\mathrm{A} 1$ are indicated by dashed lines. The corresponding injection sites are reproduced in the left column as shaded areas on a surface view of both brain hemispheres. LGNds and cortical injections were ipsilateral (top, middle) or contralateral (bottom) to the enucleated side. Tracers were injected 13 (top), 17 (middle) and 77 days (bottom) after eye enucleation

of HRP (75 and 146 days after enucleation, see Fig. 1) it was found that the retrogradely labelled neurones in the contralateral visual cortex were homogeneously distributed similar to the situation seen in nonoperated animals (Innocenti 1980). Finally, tritiated aminoacids were injected in 9 experiments into the contralateral LGNd at various times after eye enucleation ( 0 days to 24 weeks) and the results were the same as those seen in normal cats. When injected into either lamina $A$ or A1, the tracer produced a patchy pattern mainly in layer 4 of areas 17 and 18. A continuously labelled band was found, when injections comprised both layers A and A1 (not shown here).
Our findings confirm the earlier report of Singer et al. (1977) that immediately after eye enucleation, the number of neurones retrogradely labelled with HRP decreases considerably in the deafferented lamina of the LGNd contralateral to enucleation. In addition our experiments demonstrate that, 2-3 weeks after enucleation the deafferented lamina ipsilateral to enucleation is also considerably affected. In the contralateral LGNd, the proportion of labelled neurones further decreases to about $16 \%$ during the third month after enucleation. This coincides in time with the appearance of clear signs of transneuronal degeneration in the deafferented lamina (Cooke et al. 1951; Guillery 1973). The reason for the slight increase in the proportion of labelled neurones seen thereafter in the deafferented lamina remains unclear. It is possible that the axonal transport systems in geniculocortical axones has recovered to some extent, since the transneuronal atrophy has come to an end about 2-3 month after eye enucleation (Cooke et al. 1951; Guillery 1973). Alternatively, differences in age between animals could play a role (Guillery 1973). The latter conclusions should be regarded as tentative only because the number of cases with long survival (2) is certainly too small to exclude interindividual variability.

The elimination of afferent activity to one LGNd-lamina seems to impair neither anterograde transport in geniculocortical axones arising in this lamina, nor retrograde and anterograde transport in corticofugal projections back to this lamina, nor anterograde and retrograde transport in commissural projections. This statement must be qualified by the facts that we tested the retrograde transport in corticogeniculate axones only 20 days after enucleation, and that we did not analyze our autoradiographic material quantitatively.

Optic tract section leads to a delayed onset of cellular shrinkage in the LGNd as compared to that occuring after eye enucleation, at least during the first year after this operation (Cragg 1971; Garey et al. 1976). From this finding, some degree of competition between cells of the laminae related to the ipsilateral and contralateral eye respectively has been suggested to occur in the adult cat (Garey et al. 1976). The fact that axonal transport after eye enucleation in corticogeniculate and commissural axones seems to be unaffected, could be explained by the lack of binocular competition in these projection systems. Most cells of origin of the corticogeniculate projection are binocularly driven (Harvey 1980, see also Gilbert 1980) and their axones are not related to one particular lamina of the LGNd (Guillery 1967). Similarly most 
cells of origin of the commissural projection are binocularly driven (Harvey 1980) and do not form patchy projections (Sanides and Albus 1980).

Acknowledgements. We thank C. Sanides-Buchholtz for skillful technical assistance. M.-J. Pinilla-Morillo was supported by a grant from the Consejo Superior de Investigaciones Cientificas and the Max-Planck-Gesellschaft.

\section{References}

Cook WH, Walker JH, Barr ML (1951) A cytological study of transneuronal atrophy in the cat and rabbit. J Comp Neurol 94:267-291

Cragg BG (1971) The fate of axon terminals in the visual cortex during transsynaptic atrophy of the lateral geniculate nucleus. Brain Res 34:53-60

Enerbäck L, Kristensson K, Olsson T (1980) Cytophotometric quantification of retrograde axonal transport of a fluorescent tracer (primuline) in mouse facial neurons. Brain Res $186: 21-32$

Eysel UT, Grüsser OJ (1975) Intracellular postsynaptic potentials of cat lateral geniculate cells and the effects of degeneration of the optic terminals. Brain Res 98:441-455

Garey LJ, Fisken RA, Powell TPS (1976) Cellular changes in the lateral geniculate nucleus of the cat and monkey after section of the optic tract. J Anat 121:15-27

Gilbert CD (1977) Laminar differences in receptive field properties of cells in cat primary visual cortex. J Physiol 268:391421

Graham RC, Karnovsky MJ (1966) The early stages of absorption of injected horseraddish peroxidase in the proximal tubules of mouse kidney: ultrastructural cytochemistry by a new technique. J Histochem Cytochem 14:291-302

Guillery RW (1967) Patterns of fiber degeneration in the dorsal lateral geniculate nucleus of the cat following lesions in the visual cortex. J Comp Neurol 130:197-222

Guillery RW (1973) Quantitative studies of transneuronal atrophy in the dorsal lateral geniculate nucleus of cats and kittens. J Comp Neurol 149:423-438

Harvey AR (1980) A physiological analysis of subcortical and commissural projections of areas 17 and 18 of the cat. J Physiol 302: 507-534

Holtzman E, Freeman AR, Kashner LA (1971) Stimulation dependent alterations in peroxidase uptake at lobster neuromuscular junctions. Science 173:733-736

Innocenti GM (1980) The primary visual pathway through the corpus callosum: morphological and functional aspects in the cat. Arch Ital Biol 118:124-188

Ito M, Sanides D, Creutzfeld OD (1977) A study of binocular convergence in cat visual cortical neurons. Exp Brain Res $28: 21-35$

Sakakura H, Iwama K (1967) Effects of bilateral eye enucleation upon single unit activity of the lateral geniculate body in free behaving cats. Brain Res 6:667-678

Sanides D, Albus K (1980) The distribution of interhemispheric projections in area 18 of the cat: coincidence with discontinuities of the representation of the visual field in the second visual area (V2). Exp Brain Res 38:237-240

Schacker SM, Holtzman E, Hood DC (1974) Uptake of horseradish peroxidase by frog photoreceptor synapses in the dark and the light. Nature $249: 262-263$

Shatz CJ, Lindström S, Wiesel TN (1977) The distribution of afferents representing the right and the left eyes in the cat's visual cortex. Brain Res 131:103-116

Singer W, Holländer H, Vanegas H (1977) Decreased peroxidase labeling of lateral geniculate neurons following deafferentiation. Brain Res 120:133-137

Received September 21, 1988 / Accepted January 16, 1989 